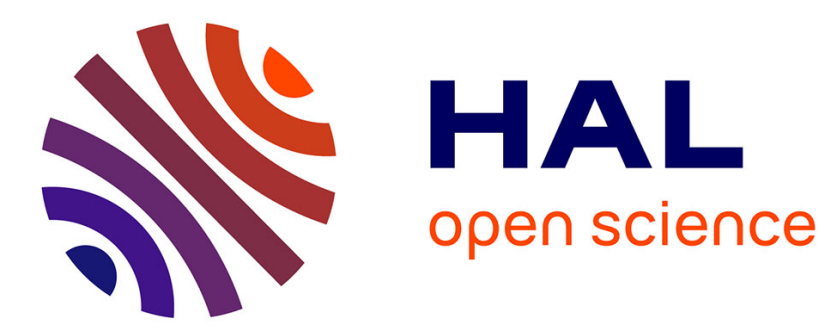

\title{
Des coups de canif dans le contrat de lecture
}

Frank Wagner

\section{To cite this version:}

Frank Wagner. Des coups de canif dans le contrat de lecture. Poétique : revue de théorie et d'analyse littéraire, 2012, 172 (4), pp.387-407. 10.3917/poeti.172.0387 . hal-02459456

\section{HAL Id: hal-02459456 https://hal.univ-rennes2.fr/hal-02459456}

Submitted on 29 Jan 2020

HAL is a multi-disciplinary open access archive for the deposit and dissemination of scientific research documents, whether they are published or not. The documents may come from teaching and research institutions in France or abroad, or from public or private research centers.
L'archive ouverte pluridisciplinaire HAL, est destinée au dépôt et à la diffusion de documents scientifiques de niveau recherche, publiés ou non, émanant des établissements d'enseignement et de recherche français ou étrangers, des laboratoires publics ou privés. 


\title{
DES COUPS DE CANIF DANS LE CONTRAT DE LECTURE
}

\author{
Frank Wagner
}

Le Seuil | « Poétique »

2012/4 n 172 | pages 387 à 407

ISSN $1245-1274$

ISBN 9782021064209

Article disponible en ligne à l'adresse :

https://www.cairn.info/revue-poetique-2012-4-page-387.htm

Distribution électronique Cairn.info pour Le Seuil.

(C) Le Seuil. Tous droits réservés pour tous pays.

La reproduction ou représentation de cet article, notamment par photocopie, n'est autorisée que dans les limites des conditions générales d'utilisation du site ou, le cas échéant, des conditions générales de la licence souscrite par votre établissement. Toute autre reproduction ou représentation, en tout ou partie, sous quelque forme et de quelque manière que ce soit, est interdite sauf accord préalable et écrit de l'éditeur, en dehors des cas prévus par la législation en vigueur en France. Il est précisé que son stockage dans une base de données est également interdit. 


\section{Frank Wagner \\ Des coups de canif dans le contrat de lecture}

\section{Contrat ou pacte?}

Fréquemment admise, et usitée, dans le domaine des études littéraires, la notion de "contrat de lecture" apparaît pourtant, à l'examen, passablement problématique $^{1}$ - du moins si par «contrat», comme y invite le Trésor de la langue française ${ }^{2}$, l'on entend un "Accord de volontés entre plusieurs personnes et faisant naître des obligations entre elles» (sens juridique), ou, a fortiori, par métonymie, un "Acte écrit constatant le contrat et destiné à en faire la preuve» (sens usuel). Il paraît en effet évident qu'en régime littéraire auteur et lecteur ne signent rien, ni ne s'engagent, au sens légal du terme, à et en rien. Entre eux, il ne saurait donc être question d'obligations stricto sensu, tant la relation consensuelle qui les unit fait la part belle à l'implicite.

Vaudrait-il mieux, dès lors, parler de "pacte" ? A cette question, les réponses du Trésor de la langue française se révèlent des plus ambiguës. Si la spécification du sens juridique ("Convention expresse ou tacite, en principe immuable, entre deux ou plusieurs parties $\left.{ }^{3} »\right)$ semble mieux correspondre aux propriétés de la relation auteurlecteur(s), la prise en compte de plusieurs sens dérivés fragilise voire invalide cette hypothèse. Ainsi, en particulier, de la définition empruntée au droit civil («Pacte sur succession future. Contrat dont l'objet est une succession non encore ouverte $»$ ), qui annule les différences potentielles entre contrat et pacte, en posant leur synonymie; ou plus encore de l'acception métonymique ("Ecrit sur lequel figure cette convention. Signer un pacte»), qui réintroduit le paramètre du document écrit, à valeur d'obligation bilatérale, validé par la signature des parties concernées.

Le pacte serait-il donc un contrat qui s'ignore? Pas tout à fait toutefois, puisque au sens le plus usuel du terme, peut-être pour partie impropre, il est d'ordinaire perçu comme moins formel, plus souple, partant moins coercitif. Aussi, à tout prendre, et même si ce distinguo n'est pas entièrement satisfaisant, il semble préférable, pour dire la nature des échanges littéraires, de parler de "pacte de lecture». Fondée sur un ensemble de conventions tacites nées de l'usage, et en l'absence de règles clairement formulées et expressément portées à la connaissance des intéressés, 
qui déclareraient solennellement y souscrire, la relation auteur-lecteur(s) relève en effet bien plutôt d'une forme de gentleman's agreement. Le "problème»- pour peu que c'en soit un - tient à ce que la qualité de gentleman n'est pas nécessairement la chose au monde la mieux partagée dans la République des Lettres, de sorte que le pacte de lecture y fait l'objet, tant en amont qu'en aval, de nombreuses transgressions - de plus en plus fréquentes au fil du temps. Ce sont précisément quelquesunes de ces infractions au pacte supposé régir la "communication» littéraire qu'il s'agira d'examiner ici, dans l'espoir de parvenir à cerner les effets et certaines des implications (notamment épistémologiques) de la relation plurivoque, souple et mobile qui s'établit entre l'auteur et ses lecteurs.

Mais, pour mieux saisir ce qui se joue à l'occasion de tels écarts, pas toujours délibérés d'ailleurs, il importe de nuancer l'une des affirmations antérieures. En effet, si le modèle juridique est impropre à rendre compte de la nature des échanges entre un écrivain et son public ${ }^{4}$, pour autant cette communication éminemment spécifique n'advient pas en dehors de toute règle, ni ne se déploie sans que la responsabilité des uns et/ou des autres s'en trouve à quelque degré engagée. En témoignent par exemple les travaux de nombre de théoriciens de la réception, à commencer par ceux de Hans Robert Jauss ${ }^{5}$, qui estime que, loin d'être totalement irrégulés, les échanges auteurlecteur(s) se développent dans un cadre déterminé, notamment par les productions littéraires antérieures. Tel est exemplairement le cas de la notion d' horizon d'attente, par laquelle l'auteur de Pour une esthétique de la réception désigne précisément, en synchronicité, un cadre de références supposé commun à l'auteur, à la critique et au public, et qui inclurait en particulier le partage de certains codes génériques. Même si la métaphore est sans doute quelque peu excessive, l'horizon d'attente pourrait donc mutatis mutandis être considéré comme la "règle du jeu» qu'est l'expérience littéraire. Mais il importe aussitôt d'ajouter que, aux yeux du théoricien de l'Ecole de Constance, enfreindre ladite règle du jeu ne constitue ni un délit ni même une attitude répréhensible: tout au contraire, cette posture «déviante», par l'introduction d'un écart esthétique, représente le moteur de l'évolution des formes littéraires - ce qui rejoint l'idée de généricité (ou de dynamisme générique), telle qu’a pu la formaliser Jean-Marie Schaeffer ${ }^{6}$. Plusieurs des exemples qui suivront témoignent bel et bien de cette aptitude indéniable de certains textes à "s'inscrire» en apparence dans un cadre générique donné, pour mieux le contester et le déborder, le faisant ainsi muter de l'intérieur - ou depuis sa périphérie, c'est selon. La fréquence et l'aisance avec lesquelles se produisent ces transgressions sont d'ailleurs aisément compréhensibles, dans la mesure où l'horizon d'attente ne désigne pas un corps de prescriptions explicites et unanimement partagées, mais un ensemble nébuleux, diffus et assez largement informulé, auquel auteurs et lecteurs souscriraient hypothétiquement. Rien de plus aisé à enfreindre qu'une règle non écrite.

Conséquemment, si ma lecture d'un texte ne constitue certes pas une activité totalement anarchique, se déployant ex nihilo, mais s'accompagne au contraire de diverses attentes plus ou moins conscientes, nées de ma fréquentation antérieure d'autres œuvres comme du consensus en vigueur dans ma communauté d'appartenance quant à ce que peut, voire doit, être tel ou tel type de texte, je ne peux pour autant nullement tenir pour assuré que l'auteur partage ces codes, ni qu'il ambitionne 
de les reconduire à l'identique, à quelques menues variantes près. L'inverse, bien sûr, est tout aussi indéniable, l'auteur ne pouvant tout au plus que spéculer sur la nature des attentes nourries par chacun des lecteurs singuliers qui composent son lectorat potentiel. En outre, les phénomènes de dépragmatisation du texte littéraire, liés à l'écart historique susceptible de séparer écrivains et publics, viennent encore considérablement accentuer ces caractéristiques. Ainsi, la «communication» littéraire pourrait-elle être définie comme un dialogue de sourds, mené en aveugle(s), sur la base de règles non écrites, évanescentes et évolutives - ce qui, loin d'être désespérant, offre une gratifiante latitude aux "partenaires» qu'elle met aux prises, et assure ainsi aux textes une relance potentiellement infinie, au fil des repragmatisations successives dont ils peuvent faire l'objet.

\section{Renversements / insuffisance / absence du pacte}

Si la notion de genre permet déjà de clarifier quelque peu la nature des relations unissant un auteur et ses lecteurs, tel est a fortiori le cas du statut des textes, c'est-àdire de leur appartenance à la fiction ou à ce que Gérard Genette propose de nommer la diction ${ }^{7}$. Pour s'en tenir à un exemple simple, certes bien loin d'épuiser le champ des possibles en la matière, on peut raisonnablement supposer que, confronté à un roman, le lecteur s'attend à y découvrir le récit d'une histoire imaginaire; en présence d'une autobiographie, à y prendre connaissance du récit authentique, du moins soucieux de véridicité, que l'auteur y fait de sa propre vie. Mais, on le constate aussitôt, bien plus que dans le cas de la simple appartenance générique, les pseudonormes qui sous-tendent ces attentes sont susceptibles d'être subverties, de façon plus ou moins volontaire, et plus ou moins perturbante pour les lecteurs - sans même évoquer, pour l'instant, leur évolution au fil du temps.

Le cas de figure le plus extrême est celui de la supercherie ${ }^{8}$, qui, eu égard à la problématique envisagée, peut se développer selon deux directions diamétralement opposées. D’une part, l'auteur peut présenter comme authentique ce qui est en fait imaginaire. Parmi de multiples autres exemples, que l'on pense à Wilkomirski ${ }^{9}$, prétendant témoigner ès qualités de rescapé des camps de la mort, alors qu'il n'en était rien. Sans même insister sur le scandale éthique suscité par une telle tromperie, la rupture du pacte de «factualité" (si l'on ose dire) pourrait suffire à provoquer l'ire des lecteurs. Ainsi en va-t-il plus généralement des écrits des mythomanes de tout poil, où l'affabulation se pare des atours de la diction. D'autre part et à l'inverse, l'auteur peut présenter comme imaginaire ce qui est authentique. On ne compte plus les exemples de particuliers qui, estimant se reconnaître sous les traits de tel ou tel prétendu personnage de "roman", poursuivent en diffamation l'auteur de ce qu'ils perçoivent comme une diction imparfaitement maquillée en fiction - de sorte que la rupture du pacte trouve en l'occurrence un prolongement sur le terrain judiciaire. On remarquera au passage que, si ces deux types de supercherie exposent leurs 
auteurs, jugés coupables de pratique (d'écriture) frauduleuse, à des poursuites pour tromperie sur la marchandise, une différence substantielle les sépare toutefois: dans le premier cas, c'est - jusqu'au moment, du moins, où elle peut être invalidée - la perfection du travestissement qui est incriminée; au lieu que, dans le second, c'est en revanche son imperfection (son insuffisance) qui est dénoncée. Jusque dans les infractions dont il fait l'objet, le pacte d'écriture-lecture autorise ainsi la mise au jour de subtiles variations de dosage.

En outre, ces différences de degré et/ou d'accent permettent également de distinguer pratiques d'écriture «illicites» et "licites». Nul ne s'insurge ainsi qu'un romancier puise dans son expérience personnelle pour élaborer ses fictions; tout au contraire, c'est même un truisme critique que la part autobiographique de nombre de romans, et une qualité (?) aussi prisée par les médias que plébiscitée par le public - alors même qu'on pourrait estimer que la pureté générique s'en trouve quelque peu altérée. Quant aux silences, déformations et embellissements dont se rendent quotidiennement coupables les autobiographes dans la relation de leur existence, sauf exagération ou omission par trop criante, ils sont le plus souvent perçus comme autant de péchés véniels, et leurs auteurs absous.

Dans les exemples de supercheries qui viennent d'être évoqués, la transgression plus ou moins clairement avérée du pacte incombait au seul auteur. Or, divers cas limites existent, qui impliquent une forme de partage des responsabilités et mettent en lumière la ductilité de la relation auteur-lecteur(s), en même temps que la fragilité des critères sur lesquels elle se fonde. Ainsi du célèbre - dans les rangs des théoriciens de la littérature s'entend - Marbot de Wolfgang Hildesheimer ${ }^{10}$. Pour mémoire, ce texte consiste en une biographie de Sir Andrew Marbot, critique d'art anglais du début du XIX ${ }^{e}$ siècle, dont nous sont notamment rapportées les rencontres avec Goethe, Schopenhauer, de Quincey, Delacroix, etc. Précision utile: contrairement aux hommes illustres dont les noms précèdent, Sir Andrew Marbot n'a jamais existé... ailleurs que dans l'imagination de son auteur, et de ses lecteurs. Plus que le procédé sur lequel il repose (accréditation d'un personnage fictionnel par mention de contacts supposés avec diverses personnes à l'existence historique avérée), ce qui rend ce cas curieux et digne d'intérêt est la position de l'auteur: si l'on en croit les déclarations rétrospectives de Hildesheimer, après la découverte de ce qui fut perçu comme le "pot aux roses", il n'a nullement voulu produire une supercherie, ni même une mystification ${ }^{11}$. Pourtant, ses premiers lecteurs ont presque unanimement lu le livre comme une biographie authentique. Dès lors, à qui la «faute»? Ce que l'on constate à la lecture de l'ouvrage est en tout cas l'absence d'indices évidents de fictionalité. De plus, la quatrième de couverture, en dehors d'une formule à double entente, mais surtout significative selon moi pour qui connaît déjà le statut fictionnel de Marbot ("Ceux qui n'ont encore jamais entendu parler d’Andrew Marbot auront du mal à comprendre comment un personnage aussi étonnant de l'époque romantique a pu rester aussi longtemps méconnu»), non seulement ne clarifie pas la donne, mais contribue au contraire à l'obscurcir, en mentionnant parmi les précédents écrits de l'auteur une biographie de Mozart. Les lecteurs sont ainsi vivement incités à inférer l'existence réelle de Sir Andrew Marbot.

On sait que la paternité du péritexte est bien souvent plurielle, et, à la rigueur, 
peu importe qu'elle incombe en l'occurrence à l'auteur, à l'éditeur, ou aux deux à la fois, puisqu'il ne s'agit ni d'instruire un procès ni même de blâmer qui que ce soit. L'intérêt théorique de ce cas particulier réside plutôt dans le constat suivant: la seule alliance du texte et du péritexte ne suffit pas ici à poser les bases d'un pacte de fictionalité, surtout compte tenu du poids de l'horizon d'attente générique (la biographie). Pour dissiper toutes les ambiguïtés et conclure le pacte (fictionnel, donc) approprié à l'ouvrage, il fallut attendre l'intervention épitextuelle de l'auteur. Que l'on souscrive ou non à l'hypothèse de la bonne foi de Wolfgang Hildesheimer, ce que nous enseigne le destin tortueux de son Marbot, outre l'importance de l'horizon d'attente, tient donc pour l'essentiel aux conséquences potentiellement fourvoyantes d'une spécification insuffisante du pacte d'écriture-lecture.

Autre cas célèbre, les Lettres portugaises ${ }^{12}$ font surgir des difficultés similaires et permettent de tirer des enseignements voisins, du moins pour partie. Christine Montalbetti rappelle en effet que, dès sa parution en 1669, le statut (référentiel ou fictionnel) de ce texte a donné matière à discussion, et "est demeuré incertain pendant des siècles ${ }^{13}$ ", avant la découverte tardive du privilège d'auteur contenant le nom de Guilleragues ${ }^{14}$. Ceux qui voyaient dans ce texte une traduction française de la correspondance amoureuse d'une authentique religieuse portugaise sont ainsi contraints de convenir qu'il s'agissait en fait d'un roman épistolaire. Souvent convoqué par les théoriciens pour démontrer l'indiscernabilité du fictionnel et du référentiel sur la base exclusive des propriétés formelles du texte, cet exemple nous renseigne aussi simultanément sur la nature du pacte littéraire, puisque le diagnostic de fictionalité (ou non) dépend à la réflexion de la prise en compte d'un contexte pragmatique. Aussi longtemps qu'un tel contexte n'est pas spécifié, les diagnostics contradictoires, fondés sur la valorisation de tel (les cinq lettres de la religieuse, évoquant les cinq actes de la tragédie: suggestion de fictionalité?) ou tel (le style désordonné et les contradictions internes: gages d'authenticité ?) aspect du texte, relèvent d'une décision herméneutique, toute subjective. Ce n'est que la découverte du nom d'auteur, élément contextuel, qui permet de résoudre ce dilemme, en favorisant l'institution d'un pacte de lecture conforme au statut fictionnel du texte, par là même et seulement alors élucidé. Autant sinon davantage encore que le Marbot de Hildesheimer, les Lettres portugaises de Guilleragues nous renseignent ainsi sur la fragilité du pacte d'écriture-lecture: qu'un seul nom (celui de l'auteur) nous manque, et tout est perturbé.

Cette nécessité du contexte pragmatique pour la mise en place d'un pacte de lecture approprié se voit spectaculairement confirmée par un autre cas d'école, les Images de Philostrate. Si, cette fois, un nom d'auteur nous est bien parvenu, à partir de l'examen de son seul texte, consistant en un enchaînement de descriptions de tableaux destinées à un enfant, il est impossible de trancher entre fictionalité et référentialité - c'est-à-dire entre l'hypothèse d'une fiction didactique et celle de l'évocation fidèle de fresques authentiques. Seule l'exhumation, désormais fort compromise, de l'hypothétique référent de cette suite d'ekphrasis aurait pu permettre de dissiper cette ambiguité. En l'absence d'un tel paramètre, force est de convenir que, même dans l'acception considérablement assouplie qui a cours pour dire les rapports de l'auteur et de ses lecteurs, un tel texte n'institue pas de pacte, 
de sorte que les hypothèses contradictoires (fiction versus diction) pourront indéfiniment être renvoyées dos à dos.

Quels enseignements pouvons-nous tirer de ce hâtif survol de quelques cas d'espèce?

- Principalement l'importance considérable du pacte dans l'économie de notre réception, puisque, de propos plus ou moins délibéré, son renversement (les diverses supercheries), son insuffisance (Hildesheimer, Guilleragues) ou son absence (Philostrate) contrarient la détection du statut des textes auxquels nous sommes confrontés, partant compromettent l'adoption d'une posture ad hoc.

- Mais aussi la diversité des ressources qui concourent à l'établissement du pacte, puisque, sur fond de conditionnement de nos lectures par l'horizon d'attente, est apparu en creux le rôle d'importance variable selon les cas joué par le texte, le péritexte, l'épitexte et le hors-texte.

- Enfin, dans la mesure où tout pacte se définit par la relation qu'il institue entre ceux qui le ratifient, le rôle crucial joué en la matière, en régime littéraire, par l'auteur et le lecteur.

Or, si, comme on l'a vu, les entorses au pacte littéraire sont fréquemment le fait de l'auteur, cela ne signifie pas que la responsabilité du lecteur soit toujours hors de cause. En effet, les dysfonctionnements d'une relation d'écriture-lecture pourtant soigneusement "verrouillée» en amont sont parfois le fait de l'instance réceptrice. Ainsi, à la périphérie du domaine qui nous occupe, le premier exemple qui vient à l'esprit est-il celui de ces spectateurs populaires qui, nous dit la légende, une fois le spectacle achevé, attendaient le traître de mélodrame à la sortie du théâtre pour lui faire un mauvais parti. Dans la mesure où les vecteurs d'immersion propres à l'art dramatique, a fortiori à la représentation scénique, diffèrent de ceux qui ont cours dans la fiction narrative, $l u e^{15}$, un tel exemple ne ressortit certes pas directement à notre propos, mais il exemplifie en revanche un paradigme qui peut $y$ avoir cours : prendre pour réel ce qui est imaginaire. Dans le champ de la lecture littéraire, la confusion du fictionnel et du référentiel correspond bien à une mauvaise compréhension ou interprétation du pacte que l'auteur entendait conclure, donc à sa rupture unilatérale et involontaire - du moins dans la plupart des cas - par le lecteur. "Quichottisme» et "bovarysme » sont d'ailleurs deux désignations notoires de ces dérives lectorales depuis longtemps déjà identifiées ${ }^{16}$. Toutefois, même si elles sont communément assimilées, les postures exemplifiées par ces deux personnages illustres mériteraient d'être distinguées. Ainsi don Quichotte ${ }^{17}$, sous l'influence des romans de chevalerie, prend-il le réel pour l'imaginaire, par exemple des moulins pour des géants; au lieu qu'Emma Bovary ${ }^{18}$ semble prendre l'imaginaire pour le rée ${ }^{19}$, par exemple les amours tourmentées des héros de fiction pour des relations interpersonnelles authentiques, à ce titre reconductibles dans sa propre existence à l'état de modèles. Mais ces nuances peuvent en effet paraître de peu d'importance en regard du travers commun aux deux personnages, qui consiste à oublier le statut fictionnel de leurs lectures pour en dégager des règles de vie, avec l'issue désastreuse que l'on sait. L'un des mérites des romans de Cervantès et de Flaubert réside ainsi dans la façon dont, par l'intermédiaire de leur protagoniste respectif, ils dialectisent une mécompréhension du pacte de lecture littéraire, dont ils favorisent au moins potentiellement par contraste le respect, à un autre niveau. Instruits par les 
contre-exemples intradiégétiques du chevalier à la triste figure et d'Emma Bovary, les lecteurs réels, hors diégèse, sont incités à ne pas prendre (plus longtemps?) les vessies fictionnelles pour des lanternes référentielles. Les ressources de la mise en abyme semblent par là même placées au service d'une efficace propédeutique, visant à prémunir le lecteur contre d'hypothétiques entorses au pacte institué par l'auteur.

Mais, là encore, précisions et nuances s'imposent. Tout d'abord, il paraît fort probable que, sauf pathologie avérée du lecteur, de telles réceptions aberrantes sont vouées à demeurer fort rares ${ }^{20}$, puisque d'ordinaire (et sauf supercherie), ressources épitextuelles, péritextuelles, métatextuelles et textuelles concourent à la clarification du statut du texte, et facilitent donc l'adoption d'une posture de réception appropriée. Ce n'est guère, on l'a vu, que lorsque les indices de ce statut font défaut, partant lorsque le pacte est insuffisamment clair, que surviennent les risques d'erreurs quant à la nature fictionnelle ou référentielle du texte. Saufpathologie, disaisje: si le rapport à la fiction implique une suspension volontaire et momentanée de l'incrédulité ${ }^{21}$, croire spontanément, sérieusement et durablement, c'est-à-dire pardelà le temps de la lecture, à ses représentations correspondrait bien à un tel rapport anormal au texte - comme tel appelé à demeurer exceptionnel et rarissime.

Toutefois, lorsqu'il est question de la relation que nous entretenons avec les textes, la "croyance" a ses degrés non seulement en termes de durée, mais aussi d'intensité; et même lorsque le statut du texte est clairement établi, divers doutes ponctuels sont toujours susceptibles de se faire jour dans l'esprit du lecteur. Par exemple, à la lecture d'un roman, lors du repérage d'un biographème notoire de l'auteur, attribué à un personnage; ou, à l'inverse, à la lecture d'une autobiographie, lors de la détection d'un élément par trop invraisemblable. Car l'horizon d'attente inclut un certain nombre d'idées reçues sur ce qu'est la littérature, certes, mais aussi sur ce qu'est la vie, comme sur le type de relations mimétiques qui peuvent ou non se développer entre ces deux pôles. La question serait alors de savoir si les doutes du lecteur demeurent circonscrits à la séquence problématique, ou si, par un phénomène de contagion métonymique, ils ne risquent pas d'être étendus au statut du texte dans son ensemble. Au nom, une fois encore, des conceptions endoxales des divers genres, ces risques de contagion semblent beaucoup plus élevés dans le cas des enclaves fictionnelles en régime référentiel que dans celui des enclaves référentielles en régime fictionnel: principalement parce que la fictionalisation du référentiel est perçue comme une infraction à l'impératif de véridicité sous-jacent à ce type d'écrit, au lieu que l'emprunt au réel en contexte fictionnel est charitablement versé au compte d'intentions mimétiques, en phase avec les aspirations au vraisemblable que la doxa prête volontiers au romancier. On sait d'ailleurs que nombre d'auteurs contemporains, à commencer par Alain Robbe-Grillet, se sont ingéniés à déjouer cette tenace stéréotypie, tantôt en instillant d'évidents biographèmes dans leurs romans, tantôt en interpolant de non moins évidentes séquences fictionnelles dans leurs écrits autobiographiques. Mais, ces exemples de transgression auctoriale délibérée mis à part, il semble bien que le lecteur puisse à tout moment être conduit à s'interroger quant au statut du texte qui lui est proposé, donc quant à la fiabilité du pacte qu'il est censé ratifier.

Encore les cas qui viennent d'être évoqués témoignent-ils d'un partage des responsabilités, puisque les soupçons des lecteurs naissent en ces occasions du repérage des 


\section{Frank Wagner}

latitudes que l'auteur prend, plus ou moins volontairement, à l'égard des "normes" supposées régir la réception de son texte. Mais on peut, différemment, envisager des cas de rupture unilatérale du pacte par le lecteur, en vertu d'une décision de principe. Il s'agirait donc alors, de propos délibéré, de lire le texte à contre-statut. Sans doute ce cas de figure ne correspond-il plus au régime courant de la lecture, et relève-t-il plutôt d'un geste critique, voire métacritique, sur le modèle des renversements paradoxaux qui ont assuré la notoriété de la critique interventionniste de Pierre Bayard. Après Et si les xuvres changeaient d'auteur? 22, la formule serait donc ici Et si les auvres changeaient de statut? ${ }^{23}$, inversion de perspective également riche en possibilités d'enseignements épistémologiques. Lire volontairement un texte fictionnel comme s'il s'agissait d'un texte référentiel, et vice versa, permet en effet de réfléchir aux propriétés de ces deux statuts, aux échanges qu'ils/elles entretiennent, aux éventuels cas de syncrèses, comme à la nature de la relation qui se met en place entre les diverses composantes de l'expérience littéraire - à commencer par l'auteur et le lecteur.

Précisons toutefois que, à y regarder de plus près, de telles lectures à rebroussestatut ne sont pas l'apanage exclusif de quelques théoriciens férus d'expérimentation, mais peuvent également se rencontrer, toutes choses égales par ailleurs, dans le cadre de pratiques de réception plus "naïves» - ce qui nous renvoie, du moins pour partie, au point précédent de cette réflexion. Ainsi m'a-t-il été donné, à l'occasion d'un colloque consacré à la voix narrative, d'assister à une communication dont l'auteur s'est ingénié, trente minutes durant, à qualifier L'Arc-en-ciel de la gravité24 - que je considérais, et considère toujours sans hésitation, comme un roman - $\mathrm{d}$ ' « autobiographie de Thomas Pynchon». Bel exemple de rupture unilatérale du pacte littéraire, à des fins «argumentatives" - disons plutôt "persuasives ». On peut aussi faire l'hypothèse que certaines des réactions indignées suscitées par Les Bienveillantes ${ }^{25}$ de Jonathan Littell procèdent d'une occultation du statut fictionnel d'un texte qui, pour traiter de réalités historiques, n'en revendique pas moins avec clarté, aussi bien épitextuellement que péritextuellement ou textuellement, la qualité de roman. Mais il est vrai qu'ici, comme dans les scandales régulièrement provoqués par les romans de Michel Houellebecq ${ }^{26}$, autant voire davantage que la «méprise» sur le statut du texte, ou que son "oubli», c'est très probablement la volonté de défendre une hypothétique exemplarité de la fiction qui motive les rejets. Pour clore cette série d'exemples sur une note plus légère, L'Equipe du jeudi 18 octobre 2012 rapporte l'initiative d'un libraire de Glasgow qui, au vu des preuves accablantes de dopage réunies par l'USADA (United States Anti-Doping Agency: Agence américaine antidopage) à l'encontre de l'ancien coureur cycliste Lance Armstrong, "a fait sa pub en rangeant l'autobiographie de l'ancien coureur au rayon "fictions»» (p. 7); geste à plus d'un titre digne d'intérêt. Tout d'abord, on observera que, excédant la pratique aujourd'hui dominante du solitaire «tête-à-texte», la «lecture» se fait ici geste public, relayé par les médias en raison de son ingéniosité. Ensuite, la classification de l'autobiographie de Lance Armstrong au rayon "fictions» de la librairie relève certes d'une décision herméneutique, mais motivée contextuellement: c'est parce que les accusations de dopage pesant sur le cycliste sont désormais avérées que le libraire se sent fondé à en inférer une plus générale attitude mensongère. De la rupture par le champion du pacte que suppose l'éthique sportive, le libraire-lecteur infère celle du pacte 
autobiographique censé sous-tendre Il n'y a pas que le vélo dans la vie ${ }^{27}$. Autrement dit, si Armstrong a pendant tant d'années menti aux médias et aux enquêteurs des agences antidopage, il en est allé de même, au moins par omission, dans le cadre de son écrit, qui dès lors déroge à l'impératif de véridicité dont, comme Philippe Lejeune ${ }^{28}$, notre libraire fait dépendre l'autobiographie en tant que genre. D’où la relégation du livre hors du champ des écrits référentiels, dans les eaux de la fiction.

Toutefois, pour savoureux qu'il soit, le geste du libraire écossais ne s'en trouve pas moins fragilisé par une approximation théorique, dans la mesure où il procède d'une assimilation du mensonge et de la fiction. Or, comme l'ont notamment démontré les travaux de Margaret Macdonald ${ }^{29}$, les assertions fictionnelles, si elles ne peuvent bien sûr être tenues pour vraies, ne sont pas davantage fausses, c'est-à-dire erronées ou mensongères, et ne correspondent pas non plus à la dynamique de l'hypothèse. Cruciale pour quiconque souhaite réfléchir à la nature du pacte littéraire, la question du statut logique de l'énoncé de fiction implique donc que, après beaucoup d'autres, on en dise deux mots. D'accord sur ce point avec Christine Montalbetti ${ }^{30}$, il me semble que la notion la plus opératoire pour cerner ce statut éminemment particulier est celle de simulation. Déjà au cœur de la définition par Coleridge de la «foi poétique» comme «suspension momentanée et volontaire de l'incrédulité », présente sous des aspects variés dans les travaux de la plupart des philosophes du langage, on la retrouve dans les réflexions anthropologiques de Jean-Marie Schaeffer, sous la forme de ce que l'auteur de Pourquoi la fiction? nomme une expérience de «feintise ludique partagée ${ }^{31}$ ». Qu'il y ait dans la production d'énoncés fictionnels assertion feinte, sans intention de tromper ${ }^{32}$, soit; mais pour rendre compte de la nature bilatérale de l'expérience littéraire, la notion de partage introduite par Schaeffer paraît essentielle ${ }^{33}$. En effet, si, produisant de tels simulacres d'assertions, l'auteur n'ambitionne nullement de me leurrer, jouer le jeu de la fiction suppose qu'à mon tour, en posture de réception, j'adopte une attitude similaire; c'est-à-dire que je fasse comme si ces énoncés "assertifs" n'étaient pas feints, tout en sachant qu'ils le sont. D'où un état mental sinon clivé, du moins biplanaire ${ }^{34}$, que nous adoptons d'ordinaire aisément, sans même y penser, sous l'influence de multiples indices valant, une fois encore, « règle du jeu» - ce que Schaeffer désigne comme "l'instauration d'un cadre pragmatique approprié à l'immersion fictionnelle ${ }^{35}$ \%. Par conséquent, une telle définition de la fiction ne se cantonne pas à la seule intentionnalité de l'auteur, mais inclut «le statut communicationnel de l'œuvre» (ibid.). En effet:

Il ne suffit pas que l'inventeur d'une fiction ait l'intention de ne feindre que "pour de faux", il faut encore que le récepteur reconnaisse cette intention et donc que le premier lui donne les moyens de le faire. C'est pour cela que la feintise qui préside à l'institution de la fiction publique ne doit pas seulement être ludique, mais encore partagée. Car le statut ludique relève uniquement de l'intention de celui qui feint: pour que le dispositif fictionnel puisse se mettre en place, cette intention doit donner lieu à un accord intersubjectif (p. 147).

De fait, lorsque l'auteur ne donne pas à son (ses) lecteur(s) les moyens de détecter le caractère ludique de l'intentionnalité qui préside à ses assertions, celles-ci risquent 
de fonctionner comme une feintise sérieuse, c'est-à-dire de leurrer les récepteurs. Tel fut, on l'a vu, le cas du Marbot de Hildesheimer, dont la nature fictionnelle passa inaperçue, faute d'un cadre pragmatique suffisamment clair pour donner matière à accord intersubjectif. Mais d'ordinaire, en situation d'immersion fictionnelle réussie, les potentiels leurres du texte sont bloqués, et requalifiés en semblants, "en quelque sorte par définition [écrit Jean-Marie Schaeffer] - c'est-à-dire par le simple fait de l'accord de feintise ludique partagée $e^{36}$ ". Egalement convaincu de l'importance d'un tel accord, je souscrirais donc volontiers pour ma part à l'idée d'un pacte de feintise ludique partagée, dont l'existence et l'utilité se trouvent démontrées a contrario par nombre des exemples précédents, cas d'espèce où son fonctionnement usuel est mis à mal. Qui dit pacte dit en effet possibilité de le déjouer, avec une intensité variable, allant de la simple entorse ludique à la transgression caractérisée. Ce sont donc quelques-unes de ces «infractions " qu'il reste à prendre en considération, en deux temps distincts; car si l'on peut certes repérer quelques cas limitrophes fort ambigus, en règle générale, la question des possibles "avenants" au pacte d'écriture-lecture connaît de substantielles variations, selon que le statut du texte relève de la fiction ou de la diction.

\section{Du côté de la fiction : jeu(x) avec le pacte}

Bien souvent, en effet, en régime fictionnel, plutôt que de se livrer à d'authentiques transgressions, il s'agit pour l'auteur, du sein du texte et/ou depuis sa périphérie, de "mettre en place un espace ludique de jeu avec la question de la fictionalité ${ }^{37}$ », auquel le lecteur se voit convié - et dont, en dépit des apparences, le pacte sort somme toute renforcé. De cette dynamique de jeu avec le pacte fictionnel participe tout d'abord, en raison même de sa dimension transactionnelle autant que transitionnelle ${ }^{38}$, le péritexte - du moins certains de ses usages paradoxaux. Des très nombreux types répertoriés par Gérard Genette dans Seuils, deux seulement seront ici retenus pour leur valeur exemplaire: celui de la préface auctoriale dénégative; celui de la préface fictionnelle ${ }^{39}$.

Dans le premier cas, l'auteur, depuis cet emplacement stratégique, renonce sur le mode déclaratif à son statut, et se présente tantôt comme simple éditeur, tantôt comme traducteur d'un manuscrit rédigé par autrui. Sous le poids de l'usage, nul sans doute n'éprouverait plus aujourd'hui la tentation de prendre de telles dénégations préliminaires au pied de la lettre, que leur stéréotypie même nous invite à lire à rebours ${ }^{40}$. «X dit a), pense non-a), et veut faire entendre non-a) à son interlocuteur. " Cette définition de l'ironie par Philippe Hamon ${ }^{41}$ s'applique parfaitement à la stratégie en cause: il n'est guère de nos jours plus sûr moyen pour un auteur de fiction d'ếtre perçu comme tel par ses lecteurs que de prétendre limiter son rôle à la découverte et/ou à la présentation d'un texte allographe. Certes, deux questions s'enchevêtrent ici: celle de la paternité du texte; celle de son statut; mais le topos 
du manuscrit trouvé apparaît désormais si connoté que sa présence dans une préface peut valoir proclamation antiphrastique de fictionalité.

Plus ambiguë est en revanche l'affirmation par l'instance préfacielle de l'authenticité de l'histoire à venir et de la réalité des personnages qui la peuplent. A elles seules, de telles assertions dénégatives (en substance: "Je déclare, et vous prie de croire, que ceci n'est pas une fiction») ne sauraient constituer un paramètre authentiquement discriminant, puisque on peut indifféremment les rencontrer en régime sérieux, dans les préfaces de textes référentiels ${ }^{42}$. Pour que, sur cette base, puisse être institué le pacte de fictionalité, il est donc nécessaire que ces assertions feintes soient désamorcées par des ressources contradictoires, qui les empêchent de fonctionner comme des leurres et les désignent comme semblants. Tel est bien sûr au premier chef le rôle de l'étiquette générique «roman», qui infirme par avance ces affirmations de non-fictionalité. De même, bien qu'à un degré moindre, une insistance exagérée de l'instance préfacielle sur l'authenticité de l'histoire racontée, nonobstant ses possibles invraisemblances, peut revêtir une valeur de signal d'alarme, en éveillant (à dessein) la suspicion des lecteurs - parmi d'autres nombreuses ressources, comme l'onomastique, l'intertextualité 43 , etc. Puisque l'ironie constitue par définition une "communication à haut risque $^{44}$ ", quels que soient les moyens élus, l'objectif réside, via la constitution d'un réseau indiciel suffisamment dense, dans la neutralisation des leurres dénégatifs, c'est-à-dire dans leur blocage à des fins de clarification indirecte du statut du texte, donc du mode de réception qu'il appelle. Et, de nouveau, l'horizon d'attente tient sa partie dans la dissipation de ces possibles ambiguïtés préfacielles: évoquant la multiplication aux XVII ${ }^{\mathrm{e}}$ et XVIII ${ }^{\mathrm{e}}$ siècles, dans La Vie de Marianne, Les Liaisons dangereuses, Les Lettres persanes, ou encore La Nouvelle Héloïse, des préfaces fictionnelles "qui présentent les fictions qu'elles introduisent comme des textes référentiels ${ }^{45}$ ", Christine Montalbetti signale à juste titre qu'il s'agit également là de «conventions du roman épistolaire ou du roman-Mémoires ${ }^{46} »$. Le caractère potentiellement fourvoyant de telles préfaces serait ainsi neutralisé par le poids des attendus hypogénériques, et relèverait dès lors d'une indication paradoxale et ludique de fictionalité.

Des divers types que Genette regroupe sous l'appellation de "préfaces fictionnelles ", celui où la part de la fiction et du jeu (avec ladite) est la plus évidente consiste en la feinte attribution de la préface à l'un des personnages de l'histoire à laquelle prélude ce seuil péritextuel, comme dans Histoire de Gil Blas de Santillane d'AlainRené Lesage ${ }^{47}$. Il n'y a bien sûr dans ce comble de la mimèsis formelle nulle tentative de supercherie, mais tout au contraire, puisque le nom d'Alain-René Lesage en première de couverture suffit à remplir une fonction de garde-fou ou de guideâne, une forme d'insistance ironique sur la fictionalité du texte, ainsi obliquement dialectisée. En la matière, la préface fictionnelle, «fiction de préface ${ }^{48}$ », ne fait guère selon Genette que systématiser l'un des traits récurrents de la préface sérieuse, et par là même le désigne au moins potentiellement à l'attention des lecteurs. C'est donc à la fois l'essence même de la fiction littéraire et l'accord intersubjectif qu'elle présuppose qui se trouvent de la sorte mis en lumière. Telle est d'ailleurs selon moi la principale fonction des préfaces fictionnelles: engager une réflexion en acte, certes ludique, sur le statut des textes qu'elles introduisent, et du même coup souligner par l'absurde la nature du pacte unissant auteurs et lecteurs. 
Or, Genette signale en outre que "cette autoreprésentation [de la préface en général, et de la préface fictionnelle en particulier] est aussi, très éminemment, celle de l'activité littéraire en général» (ibid.). De fait, il n'y a pas lieu de borner cette dialectisation indirecte du pacte d'écriture-lecture au seul péritexte, tant il est indéniable que sa prise en compte métatextuelle s'effectue aussi voire surtout du sein même du texte. Il semble au contraire logique d'estimer que la fictionalisation du péritexte résulte bien plutôt d'une extension ou d'un déport du métatextuel vers les marges du volume-livre ${ }^{49}$. Aussi est-ce sans surprise que l'on relèvera au sein même des textes le procédé de dénégation déjà évoqué: c'est d'ailleurs là un topos de la littérature réaliste, et plus encore populaire, que l'opposition déclarative à la fiction romanesque. La formule pourrait en être, à propos d'un personnage donné: "Il pensait que cela n'arrive que dans les romans... mais il n'était pas dans un roman." De telles incidentes, par exemple récurrentes chez Simenon ou Agatha Christie, tendent à l'évidence à la production contrastive de vraisemblance; mais le vraisemblable n'est, de façon notoire, que ce qui semble vrai et ne l'est donc pas. Ces formules dénégatives, de surcroît figées par l'usage, n'ont donc nullement vocation à constituer des leurres, mais de simples semblants et contribuent dès lors à désigner à rebours la nature du texte où elles figurent, comme celle du pacte (fictionnel) qui les sous-tend - puisque l'attention du lecteur est susceptible de se voir attirée par ce qui relève tout de même de la contradiction dans les termes: comment croiraisje le roman qui (m')affirme qu'il n'est pas un roman ${ }^{50}$ ?...

La dénudation du medium littéraire et du pacte sous-jacent n'est certes ici que ponctuelle, fort discrète et/car indirecte; mais l'on sait que nombre de romanciers en ont au contraire fait la pierre de touche de leur poétique romanesque - parfois dès lors considérée comme «antiromanesque». A bien y réfléchir, l'appellation d'«antiroman " n'est pourtant pas des plus judicieuses, puisque les textes qui participent de cette esthétique ne récusent pas tant l'appartenance au genre romanesque que certaines de ses conceptions, tout entières fondées sur le credo mimétique-référentiel et la production de l'illusion réaliste. Après Le Roman comique de Scarron et Le Roman bourgeois de Furetière, c'est à la fin du XVIII siècle, avec Jacques le fataliste et son maître ${ }^{51}$, que la pratique d'écriture que l'on gagnerait à qualifier de "métaromanesque» ou de "sur-romanesque» connaît son apothéose. En effet, le texte de Diderot, en dépit de la présence ponctuelle en son sein du procédé dénégatif ("Ceci n’est pas un roman ${ }^{52}$ ), constitue en fait une introduction en acte au paradoxe romanesque, puisque la mise en concurrence permanente de l'histoire des amours de Jacques et des commentaires métatextuels disruptifs du narrateur extradiégétique y relève d'une dialectique du maintien et de la levée de l'illusion, favorisant l'initiation du lecteur à la nature même de la fiction romanesque. D'où une possible diversification des plaisirs de lecture, puisque une telle fiction autoréflexive offre à la fois, alternativement, à ses récepteurs les satisfactions inhérentes à la feintise ludique et les gratifications liées à sa thématisation ironique. Comme l'écrit Jean-Marie Schaeffer :

D'une certaine façon, une fiction autodénonciatrice ne fait jamais que refléter en son sein la double attitude qui définit la feintise ludique partagée: immersion mimétique d'un côté, neutralisation de ses effets pragmatiques de l'autre. Quant au plaisir 
particulier que nous prenons à des fictions de ce type, il est peut-être dû au fait même de ces basculements répétés entre leurre et neutralisation consciente (Pourquoi la fiction?, p. 162, note 38).

Si une fiction n'est nullement obligée de se dénoncer mais peut fort bien se contenter de s'annoncer comme telle ${ }^{53}$, des textes comme Jacques le fataliste n'en jouent pas moins le rôle de miroir grossissant et, sous couvert de son apparente transgression, renforcent en fait le pacte de fictionalité, en même temps qu'ils l'exhibent.

On peut en dire autant de nombre de "nouveaux romans" (d'Alain Robbe-Grillet, Claude Simon et Jean Ricardou en particulier), héritiers sur ce plan de Jacques le fataliste, à la dimension ludique et ironique près ${ }^{54}$, et qui combinent mises en abyme, métalepses, commentaires métatextuels et renvois intertextuels afin d'obérer l'illusion référentielle et de provoquer un "progrès de l'intelligence [du lecteur] sur l'illusionnisme romanesque ${ }^{55}$ "; comme de certains textes oulipiens, en particulier La Belle Hortense ${ }^{56}$ de Jacques Roubaud, et Un cabinet d'amateur ${ }^{57}$ de Georges Perec, dont les dernières lignes ressemblent d'assez près à la formulation métatextuelle d'un pacte de feintise ludique, d'application rétroactive :

Des vérifications entreprises avec diligence ne tardèrent pas à démontrer qu'en effet la plupart des tableaux de la collection Raffke étaient faux, comme sont faux la plupart des détails de ce récit fictif, conçu pour le seul plaisir, et le seul frisson, du faire-semblant ${ }^{58}$.

Plus près de nous, les romans éminemment ludiques de Jean Echenoz témoigneraient eux aussi d'une métatextualisation ironique du pacte de fictionalité59. Ces quelques exemples, parmi bien d'autres, suffisent à établir que, si la définition du roman n'implique pas plus aujourd'hui qu'hier (ou qu'avant-hier) la dénonciation de sa nature fictionnelle, les ressources métatextuelles ont connu un tel essor durant la seconde moitié $\mathrm{du} \mathrm{xx}^{\mathrm{e}}$ siècle qu'elles nous sont désormais familières et - sauf cas d'espèce - ne nous paraissent plus guère "transgressives» (si ce n'est d'obsolètes stéréotypes réalistes). Aussi, plutôt qu'à l'«éducation » du lectorat, leur mobilisation sert désormais le plus souvent de base au déploiement d'une connivence entre l'auteur et ses lecteurs, dont la nature de la relation (le pacte fictionnel) est ainsi spécifiée.

En témoignent tout particulièrement certaines variantes du phénomène que

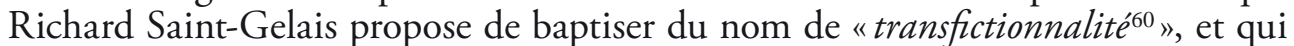
advient «lorsque deux textes ou davantage "partagent» des éléments fictifs (c'est-àdire y font conjointement référence), que ces éléments soient des personnages, des (séquences d')événements ou des mondes fictifs ${ }^{61}$ » - ce qui incite à y voir une forme de transtextualité6 6 , mais dont la particularité est d'opérer à hauteur de fiction. Ces pratiques fort diversifiées ont pour dénominateur commun d'être sous-tendues par et/ou d'enclencher une réflexion sur le statut ontologique des mondes fictionnels et de leurs habitants, qui revêt la forme d'un jeu aux accents paradoxaux; car le principe sous-jacent en est l'hypothèse d'une existence des entités fictionnelles par-delà la seule lettre des textes. Entre autres multiples exemples, une question comme "Qu'estil advenu de la fille d'Emma Bovary en dehors des pages du roman de Flaubert?» est 
par essence transfictionnelle, de même que le récit qui se déploierait ${ }^{63}$ sur cette base. S'il y a paradoxe, c'est, on le voit, parce que alors que l'existence extratextuelle des mondes et des personnages fictifs ne fait pas partie de notre univers de croyances, les littérateurs peuvent fort bien s'emparer de ce postulat pour produire de nouvelles fictions - et s'en emparent effectivement tous les jours, ou presque. Ainsi par exemple des multiples "Sherlockeries", ou continuations allographes des aventures de Sherlock Holmes, qui élisent pour point de départ l'existence réelle du Grand Détective, dont le Dr Watson serait le biographe authentique; comme des essais d'érudition holmésienne produits par les «Irréguliers de Baker Street ${ }^{64}$ ». Pour le théoricien, l'intérêt majeur de ces suites apocryphes et de ces essais critiques réside dans la façon dont le jusqu'au-boutisme holmésien et/ou holmésologique y est tempéré par une tonalité très tongue in cheeck à valeur d'indice d'ironie, qui suffit à désamorcer tout effet de leurre. Pour plus de plaisir ironique sinon pour plus de sûreté, ces transfictions établissent d'ailleurs bien souvent un réseau indiciel des plus denses, où convergent les ressources péritextuelles et métatextuelles. Tel est le cas du (au demeurant sherlockiennement très incorrect) Saigneur de la jungle de Philip José Farmer ${ }^{65}$, dont la page de faux-titre exhibe le paradoxe fondateur (Sherlock Holmes et le saigneur de la jungle y est attribué à "John H. Watson, M. D.», et seulement "présenté par Philip José Farmer, agent littéraire pour l'Amérique du Dr Watson, de Lord Greystoke, David Copperfield, Martin Eden et Don Quichotte »...), ressassé à la faveur d'une épigraphe en forme de double bind ("Tous les personnages de ce roman sont réels. Toute ressemblance avec des personnages de roman serait absolument fortuite»), d'un avant-propos réactivant le topos du manuscrit trouvé, enfin de multiples commentaires narratoriaux posant l'existence réelle de Lord Greystoke alias Tarzan. Plus que de l'hypothétique qualité du texte, le plaisir de lecture résulte ici du jeu transfictionnel, dont cette surenchère d'indices vaut exposé ludique de la règle. Il devient alors évident que, en dépit de la remise en cause dont il paraît faire l'objet, le pacte de fictionalité sort renforcé de ces transgressions feintes ${ }^{66}$. La transfictionnalité porte ainsi à son comble l'exhibition ludique et paradoxale de la relation de feintise ludique partagée sur laquelle repose toute expérience fictionnelle réussie. Par-delà l'amusement complice dont ils sont les vecteurs, les jeux transfictionnels remplissent de la sorte une fonction de révélateur épistémologique.

Des nombreux exemples qui précèdent pourrait naître la tentation de conclure que le pacte de fictionalité - sauf supercherie ou programmation insuffisante - sort en définitive indemne du traitement que lui réservent les littérateurs. Or, la situation n'est ni aussi simple ni aussi tranchée, en particulier parce que au cours des dernières décennies se sont multipliés les textes hybrides, statutairement ambigus, ne craignant pas de franchir ou de chevaucher la frontière censée séparer fiction et diction. Adepte en la matière d'une position gradualiste ou intégrationniste, Gérard Genette insiste ainsi sur la prolifération des échanges réciproques entre ces deux champs, ce qui le conduit «à atténuer fortement l'hypothèse d'une différence a priori de régime narratif entre fiction et non-fiction ${ }^{67} \%$. En effet, fréquence des phénomènes de mimèsis des formes factuelles dans la fiction, et à l'inverse des emprunts des procédés usuels des récits fictionnels dans la diction apportent " la preuve que les genres peuvent fort bien changer de normes - des normes quaprès tout [...] nul ne leur 
a imposées qu'eux-mêmes, et le respect d'une vraisemblance ou d'une "légitimité" éminemment variables, et typiquement historiques ${ }^{68}$ ».

Même si tel n'est pas l'objectif du poéticien, ses conclusions sont précieuses pour qui réfléchit à la question du pacte d'écriture-lecture, dans la mesure où, non moins que les normes génériques, avec lesquelles il a partie liée, il est également soumis à variations historiques. En attestent par exemple les nombreuses biofictions ${ }^{69}$ parues ces dernières années, en particulier celles de Jean Echenoz ${ }^{70}$. "Ravel. Roman»: le péritexte de cet ouvrage dit assez à lui seul les ambiguïtés de cette pratique d'écriture hybride, et les hésitations subséquentes de son lecteur. Si le cadre pragmatique prescrit par l'étiquette générique vaut pacte de fictionalité, il est contredit par la désignation comme protagoniste non pas d'un personnage, mais d'une personnalité historique bien connue: l'auteur du Boléro. Dès lors, le lecteur ne sait plus sur quel pied, fictionnel ou référentiel, danser. Indécision amplement confirmée par la facture même du texte, qui, s'il raconte bien (une partie de) la vie de Ravel, le fait en multipliant les procédés narratifs (focalisation interne, propos rapportés au discours indirect libre, anisochronies, etc.) et stylistiques (fluctuations pronominales, jeux de mots, métaphores incongrues, etc.) d'ordinaire perçus comme autant d'indices de fictionalité. Tout le sel de l'entreprise de Jean Echenoz, reconduite à quelques nuances près dans Courir (biofiction d'Emil Zatopek) et Des éclairs (biofiction de Nikola Tesla), provient certes de ces flottements; mais sur le plan théorique, la question du pacte apparaît ici des plus problématiques, puisque le lecteur est voué, tout au long de son activité de réception, à hésiter entre suspension momentanée et volontaire de l'incrédulité (fiction) et croyance sérieuse en le contenu narratif (diction) - position inconfortable ${ }^{71}$ : entre ces deux chaises, où s'asseoir? Parler de transgression serait peut-être excessif, mais il y a là pour le moins amendement ou avenant au pacte de feintise ludique partagée.

\section{Du côté de la diction: négociations du pacte}

A l'examen de tels textes hybrides, la frontière entre fiction et diction apparaît décidément - et pour cause - bien ténue ou poreuse; et il est fort possible quavec l'évocation de la biofiction échenozienne nous soyons en fait déjà passés de l'autre côté. En effet, si le maintien de l'étiquette générique « roman» favorise certes un jeu avec le pacte fictionnel, il n'en est pas moins vrai que Ravel, Courir et Des éclairs proposent aussi une forme de redéfinition in actu du pacte référentiel, à la faveur d'un renouvellement du genre biographique. Un pas de plus, et nous nous retrouvons sur le territoire de la diction où, plus que de jeu, c'est d'une véritable tentative de négociation du pacte, à nouveaux frais, qu'il s'agit bien souvent. En témoigneraient dans une certaine mesure, dès les années 1970, New Journalism et Nouvelle Histoire, de même que les très nombreuses "fictionalisations ${ }^{72}$ " de faits divers, de L'Adversaire d'Emmanuel Carrère ${ }^{73}$ à Sévère de Régis Jauffret ${ }^{74}$, parues ces dernières années; 
mais le cas le plus clairement emblématique de cette autre posture, plus contestataire que ludique, est certainement celui du renouvellement des écritures intimes par remise en cause (plus ou moins frontale) du pacte autobiographique. Chacun sait que si Philippe Lejeune, entre autres paramètres, fait dépendre l'existence de l'autobiographie en tant que genre de l'identité de l'auteur, du narrateur et du personnage $e^{75}$, en revanche les textes que l'on rassemble depuis lors sous la bannière controversée de l'autofiction ont en commun de déjouer l'assimilation conventionnelle de ces trois instances. Ainsi Genette évoque-t-il le " pacte délibérément contradictoire propre à l'autofiction ("Moi, auteur, je vais vous raconter une histoire dont je suis le héros mais qui ne m'est jamais arrivée") ${ }^{76}$ », qu'il illustre d' "une formule [elle aussi] logiquement contradictoire: $\mathrm{A} \neq \mathrm{N} ; \mathrm{N}=\mathrm{P} ; \mathrm{A}=\mathrm{P}^{77}$ ». Sans doute, compte tenu de la diversité des infractions empiriques au pacte autobiographique, une telle formule est-elle réductrice, et peut-on préférer distinguer, à la suite de Laurent Jenny ${ }^{78}$, les autofictions obtenues, premièrement, par « fictionalisation de l'histoire du personnage-narrateur», deuxièmement, par «fictionalisation de l'identité du narrateur ", et, troisièmement, par établissement d'une distinction fictive des identités du personnage d'une part, du couple auteur-narrateur d'autre part. Mais, en termes de pacte, ce qui importe est bien la dimension intrinsèquement contradictoire de la double postulation autofictionnelle. Comme l'a également montré Marie Darrieussecq ${ }^{79}$, si l'acte illocutoire qui sous-tend l'autofiction relève indéniablement de la contradiction, c'est que, sur ce plan, ce "genre» consiste en une assertion qui se donne à la fois comme feinte et comme sérieuse, de sorte que le lecteur ne dispose pas - puisque l'auteur ne les lui fournit pas - des moyens d'effectuer le départ entre valeur fictionnelle et/ou référentielle de ce qu'il lit. Même si les deux paramètres sont évidemment liés, plus que le refus de souscrire à la triple identité de l'auteur, du narrateur et du personnage, l'essentiel est donc en définitive ici la latitude prise par l'auteur à l'égard de l'impératif de véridicité que la tradition générique place au fondement de l'écriture autobiographique, et plus généralement des écrits référentiels.

Sur le plan théorique, l'un des intérêts de l'autofiction réside dans la façon dont cette forme favorise la mise au jour de l'historicité des codes génériques comme du pacte d'écriture-lecture qui les sous-tend. En effet, ce genre (d'ailleurs pluriel) repose sur l'hypothèse de la caducité du pacte autobiographique traditionnel : si l'on prête attention aux déclarations épitextuelles des auteurs d'autofictions, au péritexte de leurs ouvrages, ou encore aux commentaires métatextuels qui y abondent, on s'avise que, plus que d'une volonté d'expérimentation à tous crins, c'est d'une conception renouvelée du sujet et de ses rapports à l'acte d'écrire qu'est née cette nouvelle forme d'écriture intime - dont la dimension initialement perçue comme tératologique a fini par se banaliser au fil du temps, au point de constituer en quelque sorte de nos jours une norme générique de substitution. Certes, ces «mythomanies littéraires ${ }^{80}$ » témoignent d'une remarquable diversité esthétique, de «l'autobiographie de l'inconscient» d'un Doubrovsky à «l'autobiographie consciente» (c'est-à-dire consciente des apories du genre sous sa forme canonique) d'un Robbe-Grillet, mais toutes prennent acte d'une mutation épistémologique d'envergure: la conviction de l'impossibilité pour le sujet de coïncider pleinement avec lui-même, sans reste, et de se représenter sincèrement par l'écriture, sans déformations. Aussi la devise des auteurs 
d'autofictions pourrait-elle être: Le moi est mort; vive le moi! Il importe donc à leurs yeux de frayer de nouvelles voies pour dire ce retour spectral d'un sujet travaillé par le vide et le manque d'être, la représentation de soi de type autobiographique cédant dès lors le pas à la figuration de soi de type autofictionnel.

Or, en dépit des apparences, ces considérations ontologiques sont bel et bien liées à la question du pacte, dans la mesure où, depuis les années 1960, nombre de théories du sujet accordent une place centrale à la notion de fiction. Ainsi de Jacques Lacan, affirmant en 1966 que «le moi, dès l'origine, serait pris dans une ligne de fiction ${ }^{81}$ ", ou de Roland Barthes, avertissant ses lecteurs en ces termes, en ouverture de Roland Barthes par Roland Barthes: "Tout ceci doit être considéré comme dit par un personnage de roman ${ }^{82}$.» Si le moi ne préexiste pas à l'écriture sous une forme apriorique et pérenne qu'il ne resterait plus qu'à retranscrire, alors il s'agit de le forger (fingere) dans et par le geste scriptural même, dont on s'avise ainsi des capacités heuristiques. De ce travail de (re-)construction identitaire par l'affabulation témoignent par exemple $W$ ou le souvenir d'enfance de Georges Perec ${ }^{83}$, autobiographie oblique ${ }^{84}$ dont il s'agit de l'un des principes fondateurs; mais aussi Fils de Serge Doubrovsky, que sa célèbre quatrième de couverture présente comme "Fiction, d'événements et de faits strictement réels ${ }^{85}$ »; mais encore la trilogie des Romanesques de Robbe-Grillet ${ }^{86}$, où la conviction du primat de l'imaginaire dans la conscience de soi motive la fictionalisation progressivement accrue du vécu biographique de l'auteur au fil des volumes successifs. Quel que soit le rapport de ces auteurs à la psychanalyse - ambigu pour Perec, assumé pour Doubrovsky, conflictuel pour Robbe-Grillet -, l'entreprise d'autoscription à laquelle ils s'adonnent chacun à leur façon relève de ce que cette discipline nomme un travail de perlaboration.

D'aucuns objecteront que le vocable «fiction" connaît ici une extension excessive et revêt une acception trop éloignée de celle que lui attribuent d'ordinaire les théoriciens de la littérature - ce qui risquerait d'évider fâcheusement ce terme de son sens. Mais cette dérive sémantique ne constitue peut-être après tout qu'un inconvénient passager, donc mineur, dans la mesure où elle conduit en définitive bien souvent les auteurs qui la revendiquent à mobiliser dans leurs écrits intimes les ressources de la fiction au sens fort ou plein du terme - les chapitres relatifs à l'île W dans Wou le souvenir d'enfance, les scènes sado-érotiques de la Forêt des Pertes dans Angélique ou l'Enchantement, etc. L'acte illocutoire de l'autofiction n'en demeure pas moins contradictoire, et le pacte qui en découle fort ambigu, mais cela ne signifie nullement que la posture de réception ainsi présupposée serait par essence aporétique, pas plus que les textes qui la "programment» ne sauraient, sur cette base, être décrétés illisibles. Au contraire, le plaisir de lecture spécifique que dispense l'autofiction est précisément fonction du flottement ainsi institué entre fiction et diction. En outre, on peut estimer qu'auteurs et lecteurs, puisqu'ils baignent dans la même noosphère, partagent au moins jusqu'à un certain point l'épistémè contemporaine en matière de conception du sujet, de l'écriture et de leurs rapports. Si l'hypothèse paraissait trop optimiste, gageons que les multiples précisions métatextuelles dont regorgent les autofictions puissent contribuer à une redistribution efficace des cartes, à la faveur de ce qui, pour le coup, ressemble d'assez près à l'exposé d'une règle du jeu - ou, si l'on préfère, à une négociation explicite du pacte d'écriture-lecture. Bref, 
l'exemple de l'autofiction témoigne assez clairement de l'historicité des codes génériques, c'est-à-dire également de leur aptitude à évoluer au fil du temps, de pair avec les paramètres régissant la relation auteur-lecteur(s).

De ce long parcours il ressort que, si la relation de l'auteur et de ses lecteurs n'est évidemment pas régie par un contrat stricto sensu, elle n'en est pas moins conditionnée par une forme de pacte, tributaire de l'horizon d'attente et engageant à des degrés divers la responsabilité des uns et des autres, mais dont le caractère tacite, souple et mobile autorise, en amont comme en aval, de multiples accommodements d'intensité variable. En définitive, transgressions (supercheries; lectures à contre-statut), insuffisance (Marbot, les Lettres portugaises), voire absence (les Images) du pacte; comme, du côté de la fiction, constitution d'un espace de jeu avec le pacte, du côté de la diction, tentatives pour l'amender - en phase avec l'évolution historique de l'épistémè, suscitant une relative indifférenciation des statuts textuels - témoignent, quand bien même "par l'absurde", de son importance dans l'économie de notre réception, comme plus généralement dans l'expérience littéraire, dont la spécification du cadre pragmatique constitue la condition de possibilité d'un nécessaire accord intersubjectif.

\section{Université Rennes 2}

\section{NOTES}

1. Pour un ensemble de réflexions sur la notion de "contrat de lecture», voir Emmanuel Bouju (éd.), Littératures sous contrat, Rennes, Presses universitaires de Rennes, «Interférences», 2002.

2. Trésor de la langue française informatisé (http://www.atilf.atilf.fr/), entrée "Contrat», consultée le 6 octobre 2012.

3. Ibid., entrée «Pacte», je souligne.

4. Ce qui ne signifie évidemment pas que, sur un autre plan, dont il ne sera pas question ici, la littérature échapperait à tout cadre légal, hypothèse saugrenue que la notion même de droit d'auteur suffirait, s'il en était besoin, à invalider.

5. Hans Robert Jauss, Pour une esthétique de la réception (1972-1975), Paris, Gallimard, 1978 pour la trad. fr.

6. Jean-Marie Schaeffer, «Du texte au genre. Notes sur la problématique générique», dans Gérard Genette et alii, Théorie des genres, Paris, éd. du Seuil, "Points Essais», 1986, p. 179-205; Qu'est-ce qu'un genre littéraire?, Paris, éd. du Seuil, «Poétique», 1989.

7. Gérard Genette, Fiction et diction, Paris, éd. du Seuil, «Poétique», 1991. Gérard Genette précise en fait que «Le critère rhématique [...] peut déterminer deux modes de littérarité par diction. L'un (la poésie) est de régime constitutif. [...] L'autre [...] (la prose non fictionnelle) ne peut être perçu comme littéraire que de façon conditionnelle [...]» (p. 8). Dans la mesure où ma réflexion porte non pas sur la littérarité des textes, mais sur l'hypothétique existence d'un pacte d'écriture-lecture, fonction de leur statut, je prendrai donc le terme genettien de "diction" dans une acception restreinte - d'autant plus que je n'aborderai pas la question de la poésie. Il s'agira donc pour l'essentiel de désigner ainsi la "prose non fictionnelle», en évitant le recours systématique à une formulation négative.

8. Sur cette notion, comme sur ses rapports à la mystification, on se reportera à Jean-François Jeandillou, Esthétique de la mystification. Tactique et stratégie littéraires, Paris, éd. de Minuit, «Propositions», 1994. 
9. Bruno Grosjean/Dösseker alias Binjamin Wilkomirski, Fragments: une enfance, 1939-1948 (1995), Paris, Calmann-Lévy, 1996 pour la trad. fr. utilisée.

10. Wolfgang Hildesheimer, Sir Andrew Marbot (1981), Paris, éd. J.-C. Lattès, 1984 pour la trad. fr. utilisée.

11. Sur cette question, voir les observations de Jean-Marie Schaeffer, Pourquoi la fiction?, Paris, éd. du Seuil, «Poétique», 1999, p. 133 sq.

12. Texte originellement paru sous le titre Lettres portugaises traduites en français, Paris, Claude Barbin, 1669; Paris, Flammarion, 2009, "GF" pour l'édition utilisée. On remarquera que la mention des opérations de traduction dans le titre original pouvait valoir indice (trompeur) de référentialité.

13. Christine Montalbetti, La Fiction, Paris, Garnier-Flammarion, «Corpus», 2001, p. 22.

14. Gabriel-Joseph Guilleragues ou comte Gabriel-Joseph de Lavagne.

15. Sur les différents vecteurs d'immersion fictionnelle en fonction des genres et media, voir JeanMarie Schaeffer, Pourquoi la fiction?, op. cit., en particulier le chapitre IV («De quelques dispositifs fictionnels»), p. 231-315.

16. Et par exemple brièvement analysées par Christine Montalbetti, La Fiction, op. cit., p. 125 et 137.

17. Dans Miguel de Cervantès, L'Ingénieux Hidalgo don Quichotte de la Manche (1605-1615), Paris, Gallimard, 2010, «Folio Classique» pour l'édition utilisée.

18. Dans Gustave Flaubert, Madame Bovary. Mours de province (1857), Paris, Gallimard, 2001, «Folio Classique" pour l'édition utilisée.

19. Cette affirmation demanderait bien évidemment à être nuancée. Ainsi peut-on se demander si la «folie» du personnage de Cervantès ne relève pas assez largement de l'aveuglement volontaire. Quant à Emma Bovary, il semble que son erreur consiste plutôt à prêter à la fiction une exemplarité problématique, au lieu de se contenter (sagement) de ses pouvoirs de modélisation.

20. Entendons-nous: ce sont les cas de confusion durable du fictionnel et du référentiel que je considère comme «fort rares». En revanche, il me semble que notre rapport au monde est pour partie façonné par des modèles culturels dont une bonne part relèvent de la fiction - dont il importe une fois encore de distinguer capacités de modélisation (que je suis tout disposé à lui reconnaître) et exemplarité (à mes yeux problématique).

21. Selon la célèbre formule de Samuel Taylor Coleridge, "[...] willing suspension of disbelief for the moment, which constitutes poetic faith" (Biographia Literaria, 1817), The Collected Works, Princeton, Princeton University Press, 1983, t. VII, vol. 2, p. 6.

22. Pierre Bayard, Et si les œuvres changeaient d'auteur?, Paris, éd. de Minuit, «Paradoxe», 2010.

23. Tous droits réservés...

24. Thomas Pynchon, L'Arc-en-ciel de la gravité (1973), Paris, Plon, 1975 (sous le titre de Rainbow), puis Paris, éd. du Seuil, 1988, «Fiction \& Cie» pour la trad. fr. utilisée.

25. Jonathan Littell, Les Bienveillantes, Paris, Gallimard, 2006 (prix Goncourt).

26. En particulier lors de la parution de Plateforme, Paris, Flammarion, 2001, qui valut à Michel Houellebecq d'être soupçonné de faire l'apologie du tourisme sexuel.

27. Lance Armstrong, Il n'y a pas que le vélo dans la vie (2000), Paris, Albin Michel, 2000 pour la trad. fr., rééditée dans Le Livre de Poche en 2003.

28. Philippe Lejeune, Le Pacte autobiographique, Paris, éd. du Seuil, «Poétique», 1975.

29. Margaret Macdonald, «Le langage dans la fiction» (1954), Poétique, n 78, avril 1979 pour la trad. fr., reprise dans Gérard Genette (éd.), Esthétique et poétique, Paris, éd. du Seuil, «Points Essais», 1992, p. 203-228.

30. La Fiction, op. cit., p. 17.

31. Pourquoi la fiction?, op. cit., p. 145 sq. et passim.

32. John Searle, Sens et expression. Etudes de théorie des actes de langage (1979), Paris, éd. de Minuit, «Le Sens commun», pour la trad. fr., 1982, p. 108-111.

33. Idée me semble-t-il déjà présente à l'état embryonnaire chez Searle (op. cit.), avec l'hypothèse d'une nécessaire reconnaissance par le lecteur de l'intentionalité de l'auteur.

34. Pourquoi la fiction?, op. cit., p. 190. L'adjectif «biplanaire» est emprunté à Iouri Lotman, La Structure du texte artistique (1970), Paris, Gallimard, "Bibliothèque des sciences humaines" pour la trad. fr., 1973, p. 106.

35. Pourquoi la fiction?, op. cit., p. 146.

36. Ibid., p. 192, je souligne. Il est intéressant de remarquer que, citant ce passage, Christine Montalbetti remplace le substantif "accord» utilisé par Jean-Marie Schaeffer par le substantif «contrat» ( $\mathrm{La}$ Fiction, op. cit., p. 140). 


\section{Frank Wagner}

37. Christine Montalbetti, La Fiction, op. cit., p. 16.

38. Gérard Genette, Seuils, Paris, éd. du Seuil, «Poétique», 1987, p. 8.

39. Dans la typologie proposée par Gérard Genette, la préface «auctoriale dénégative» est analysée au nombre des "préfaces fictionnelles» (Seuils, op. cit., p. 255-270). Cette classification, reposant sur l'établissement d'un distinguo entre «fictionnel» et "fictif» (p. 256), est tout à fait cohérente; simplement, en les dissociant pour les besoins de mon exposé, je souhaiterais insister sur les propriétés respectives de ces types - sans pour cela minimiser la convergence de leurs fonctions. Pour la bonne intelligence de ce qui suit, reste à préciser que certaines de mes "préfaces fictionnelles» seraient donc pour Genette autant de "préfaces fictives». Dont acte.

40. Du moins lorsque le contexte pragmatique est suffisamment clarifié par le biais d'autres ressources, car il va sans dire que l'éditeur authentique d'un non moins authentique manuscrit allographe usera de formules similaires pour établir avec précision la part qui revient à chacun.

41. Philippe Hamon, L'Ironie littéraire. Essai sur les formes de l'écriture oblique, Paris, Hachette, 1996, p. 19.

42. Quoique... l'auteur d'un texte référentiel n'a en théorie pas de raison de souligner outre mesure un statut qui a priori ne souffre pas de contestation.

43. Voir par exemple Le Treizième Guerrier (1976) de Michael Crichton, Paris, Robert Laffont, 1999 pour la trad. fr., Le Grand Livre du Mois, «Best-Sellers» pour l'édition citée. Dans l'appareil critique fantaisiste de cette réécriture biaise de la saga de Beowulf, l'un des traducteurs cités se nomme "Per Fraus-Dolus", c'est-à-dire littéralement, pour les lecteurs qui n'y perdent pas leur latin, "par ruse et par tromperie» - phénomène de surmotivation onomastique qui dit assez clairement la dimension imaginaire de cette source, partant le caractère fictionnel du texte que ce «Professeur emeritus de littérature comparée à l'université d'Oslo, Norvège» (ibid.) est supposé avoir traduit. De même, la bibliographie en fin de volume contient, outre plusieurs références à des essais historiques authentiques sur les Vikings, un renvoi à "Azharedn Abdul. Necronomicon (H. P. Lovecraft éd.), Providence, Rhode Island. 1934» (p. 204), dont tout connaisseur de l'œuvre de Lovecraft est à même d'apprécier la dimension fictionnelle, avec les mêmes implications que précédemment. Ces phénomènes, dont on notera en passant qu'ils excèdent la seule préface stricto sensu pour concerner le péritexte dans son ensemble, sont également présents dans les écrits pseudoscientifiques de Georges Perec, publiés sous le titre de Cantatrix Sopranica L. et autres écrits scientifiques, Paris, éd. du Seuil, «La Librairie du xx siècle», 1991 (posthume).

44. L'Ironie littéraire, op. cit., p. 36.

45. La Fiction, op. cit., p. 15.

46. Ibid.

47. Alain-René Lesage, Histoire de Gil Blas de Santillane (1715-1735), Paris, Gallimard, "Folio Classique" pour l'édition utilisée, 2006. Dans la terminologie genettienne (Seuils, op. cit., p. 267-269), il s'agit là d'une "préface actoriale fictive", dont il donne d'autres exemples (Aventures d'Arthur Gordon Pym, Euvres complètes de Sally Mara, etc.).

48. Gérard Genette, Seuils, op. cit., p. 269.

49. Quoique, à la réflexion, déterminer en la matière quelque préséance peut paraître aussi délicat que d'établir avec assurance qui, de la poule ou de l'œuf, vit le jour avant l'autre...

50. Sans préjuger pour autant de la diversité des actualisations empiriques d'un tel effet de lecture.

51. Denis Diderot, Jacques le fataliste et son maître (1796, posthume), Paris, Librairie Générale Française, Le Livre de Poche pour l'édition utilisée, 1983.

52. Voir notamment p. 25 («Il est bien évident que je ne fais point un roman [...]») et p. 52 («[...] ceci n'est point un roman $[\ldots] 川)$.

53. Ibid., p. 162.

54. Même si jeu et ironie sont loin d'être absents de l'œuvre de Robbe-Grillet, y compris du traitement qui y est réservé aux procédés métatextuels.

55. Mireille Calle-Gruber, "Alain Robbe-Grillet ou la reprise-en-avant", Critique, n 651-652, aoûtseptembre 2001, p. 613.

56. Jacques Roubaud, La Belle Hortense, Paris, Ramsay, «Mots», 1985.

57. Georges Perec, Un cabinet d'amateur, Paris, Balland, 1979 puis 1988; Le Livre de Poche (1989) pour l'édition citée.

58. Op. cit., p. 125 et dernière, je souligne.

59. Entre autres multiples exemples, en atteste cette intervention métaleptique du narrateur extradiégétique dans Je m’en vais (Paris, éd. de Minuit, 1999; puis 2001, "Minuit Double» pour l'édition citée, 
p. 170) : «Personnellement je commence à en avoir un peu assez de Baumgartner. Sa vie quotidienne est trop fastidieuse. A part vivre à l'hôtel, téléphoner tous les deux jours et visiter ce qui lui tombe sous la main, vraiment il ne fait pas grand-chose.» Voir aussi les pages 208-209.

60. Richard Saint-Gelais, Fictions transfuges. La transfictionnalité et ses enjeux, Paris, éd. du Seuil, «Poétique», 2011.

61. «Entretien avec Richard Saint-Gelais: Fictions transfuges. La transfictionnalité et ses enjeux» (propos recueillis par Frank Wagner), Vox-poetica (http://www.vox-poetica.org/), mis en ligne le 20 avril 2012, p. 2.

62. Gérard Genette, Palimpsestes. La littérature au second degré, Paris, éd. du Seuil, «Poétique», 1982, p. 7 et passim.

63. Et s'est en fait déjà «déployé»: Claude-Henri Buffard, La Fille d'Emma, Paris, Grasset, 2001. Voir les commentaires de Richard-Saint-Gelais aux pages 27, 59, 66-68, 141, 183, 317-318, 437 et 451 de Fictions transfuges, op. cit.

64. Les BSI (pour "Baker Street Irregulars», expression désignant à l'origine dans les fictions de Conan Doyle les gamins des rues occasionnellement employés comme espions par le détective), club fondé en 1934 par Christopher Morley, constituent la première et la plus active des sociétés holmésiennes.

65. Philip José Farmer, Le Saigneur de la jungle (1974), Grenoble, éd. Jacques Glénat, "Marginalia», pour la trad. fr., 1975.

66. Pour autant, la transfictionnalité, même si elle ne vise nullement à duper les lecteurs, n'en porte pas moins une forme "d'inquiétude» (certes joueuse) sur la frontière entre le fictionnel et le référentiel, et aboutit par là même à une célébration ludique des pouvoirs de l'imaginaire.

67. Dans Fiction et diction, op. cit., p. 91.

68. Ibid., p. 93.

69. Expression proposée par Alain Buisine, «Biofictions», Revue des sciences humaines (Le Biographique), $\mathrm{n}^{\circ} 224,1991$, p. $7-13$.

70. Jean Echenoz, Ravel, Paris, éd. de Minuit, 2006; Courir, Paris, éd. de Minuit, 2008 ; Des éclairs, Paris, éd. de Minuit, 2010.

71. A vrai dire, cet inconfort concerne surtout le théoricien réfléchissant aux implications de tels textes statutairement hybrides; car, autant que je puisse en juger d'après mes propres réactions, pour le lecteur empirique, "l'hésitation» de ces récits entre biographie et roman est des plus stimulantes, et dispensatrice d'un plaisir spécifique - précisément lié à cette double «transgression ".

72. Sur la part respective du référentiel et du fictionnel dans L'Adversaire (Paris, POL, 2000) d'Emmanuel Carrère, je me permets de renvoyer à Frank Wagner, «Le "roman" de Romand", Roman 20-50, $\mathrm{n}^{\circ}$ 34, décembre 2002, p. 107-124; puis Vox-poetica.

73. Emmanuel Carrère, L’Adversaire, op. cit.

74. Régis Jauffret, Sévère, Paris, éd. du Seuil, «Cadre rouge», 2010.

75. Dans L'Autobiographie en France, Paris, Armand Colin, 1971, comme dans Le Pacte autobiographique, op. cit.

76. Fiction et diction, op. cit., p. 86.

77. Ibid., p. 87. La figure élaborée par Genette épouse la forme d'un triangle, que mes compétences limitées en informatique m'interdisent de reproduire à l'identique.

78. Laurent Jenny, "Méthodes et problèmes. L'autofiction", http://www.unige.ch/lettres/framo/enseignements/methodes/autofiction/afintegr.html Consulté le 6 octobre 2012.

79. Marie Darrieussecq, "L'autofiction, un genre pas sérieux", Poétique, n 107, septembre 1996, p. 369-380.

80. Vincent Colonna, Autofiction \& autres mythomanies littéraires, Auch, Tristram, 2004.

81. Jacques Lacan, Ecrits, Paris, éd. du Seuil, 1966, p. 94.

82. Roland Barthes par Roland Barthes, Paris, éd. du Seuil, «Ecrivains de toujours», 1975.

83. Georges Perec, W ou le souvenir d'enfance, Paris, Denoël, 1975.

84. Selon la formule de Philippe Lejeune, La Mémoire et l'oblique. Georges Perec autobiographe, Paris, POL, 1991.

85. Serge Doubrovsky, Fils, Paris, Galilée, 1977, quatrième de couverture, je souligne.

86. Alain Robbe-Grillet, Le miroir qui revient, Paris, éd. de Minuit, 1985; Angélique ou l'Enchantement, Paris, éd. de Minuit, 1988; Les Derniers Jours de Corinthe, Paris, éd. de Minuit, 1994. 